ISSN 0258-7122

Bangladesh J. Agril. Res. 35(1) : 1-10, March 2010

\title{
POPULATION DYNAMICS OF MYCOFLORA AND INCIDENCE OF BLACK POINT DISEASE IN WHEAT GRAINS
}

\author{
P. K. MALAKER ${ }^{1}$ AND I. H. MIAN ${ }^{2}$
}

\begin{abstract}
An attempt was made to monitor the prevalence of different fungi associated with floret lemma and developing grains of wheat and to assess the subsequent incidence of black point disease under different exposure periods of the spikes to airborne inocula of the causal fungi. Altogether 16 fungi representing 11 genera were detected from lemma and developing grains. The predominant fungi, in order of prevalence, were A. alternata, B. sorokiniana, C. cladosporioides, C. lunata, Fusarium spp. and E. purpurascens. Other fungi occurring less commonly were A. triticina, C. pallescens, Nigrospora sp., Phoma sp., Chaetomium sp., B. tetramera, B. oryzae, Aspergillus flavus, A. niger and Doratomyces sp. The incidence of all the fungi except Fusarium spp. was higher in lemma than in grains. In lemma, A. alternata occurred with the highest frequency, while the incidence of $B$. sorokiniana was found highest in grains. The population of A. alternata, B. sorokiniana, C. lunata, E. purpurascens and Fusarium spp. increased with the age of lemma and developing grains whereas the incidence of $C$. cladosporioides increased at early stages but declined at later stages of grain development. Other fungi did not follow any definite pattern in their incidence during grain development. The occurrence of black pointed grains per spike, percent black pointed grains and black point index were found increasing with the increase in exposure period of the spikes to airborne inocula of the causal fungi.
\end{abstract}

Keywords: Mycoflora, black point, wheat.

\section{Introduction}

Seeds are the carriers of various plant pathogenic fungi, bacteria, viruses and nematodes. When a pathogen is associated with seeds, in most of the cases, it produces a variety of symptoms on the seeds. These symptoms include seed rot, shrivelling of grain, seed necrosis and seed discolouration. Neergaard (1979) reported that many parasitic seedborne fungi infect the seed coat causing conspicuous black, brown or grey necrotic discolouration. Black point of wheat also known as kernel smudge, is characterized by brown to black discolouration mainly restricted to the embryonic end of the seed (Adlakha and Joshi, 1974). Occasionally, the symptom can be seen near the brush end, in the ventral crease,

${ }^{1}$ Principal Scientific Officer, Wheat Research Centre, BARI, Dinajpur-5200, ${ }^{2}$ Professor, Bangabandhu Sheikh Mujibur Rahman Agricultural University, Gazipur-1706, Bangladesh. 
or on any other part of the kernel. In case of severe infection, the whole grain may be discoloured and shrivelled.

Black point of wheat caused by Bipolaris sorokiniana occurs almost all over the world wherever wheat crop is grown (Mathur and Cunfer, 1993). However, it is an important disease of wheat seed crop in Bangladesh (Fakir, 1998). Black point has adverse effects on seed weight, germination and grain yield. The reduction in 1000 -grain weight was as large as $41.96 \%$ when the grains were severely infected with the black point fungi (Rahman and Islam, 1998). The germination of black point affected seeds was reduced to a great extent as compared to healthy grains (Khanum et al., 1987). However, the reduction in germination was found to be related with the severity of black point infection (Dhruj, 1991).

The fungi commonly involved in the black point disease of wheat include Alternaria alternata, Bipolaris sorokiniana, Cladosporium cladosporioides, Curvularia lunata and Fusarium spp. (Fakir et al., 1989; Dey et al., 1992). Under the favourable conditions of disease development, the airborne inocula of the causal fungi infect the spikes and ultimately lead to the development of black point on the grains. However, the characteristic discolouration of the kernels usually appears during the soft dough to hard dough stages of grain development (Talukder and Fakir, 1993; Ahmed et al., 1994).

The present study was undertaken to determine the prevalence of different fungi associated with floret lemma and developing grains and to assess the incidence of black point disease under different exposure periods of spikes to airborne inocula of the causal fungi.

\section{Materials and Method}

A black point susceptible wheat variety 'Kanchan' was grown in the experimental field of Bangabandhu Sheikh Mujibur Rahman Agricultural University (BSMRAU), Gazipur during 2000-2001 crop season. Recommended doses of manures and fertilizers were used. The seeds were sown at the rate of $120 \mathrm{~kg} / \mathrm{ha}$ in lines with $20 \mathrm{~cm}$ spacing between rows. Sowing was done in the week of November. Weeding, mulching and irrigation were done as and when necessary for maintaining normal crop growth.

After heading, samples of four hundred florets from the middle of randomly selected 100 spikes in four replicates were collected at weekly intervals for determining the incidence of the associated fungi. The sampling of florets was continued for eight weeks upto dead ripening stage. In addition, randomly selected 20 spikes in four replicates were covered with glassine paper bags during each sampling to create different exposure periods of the spikes to airborne inocula of the fungi involved in the development of black point on the 
grains. Before bagging of the selected spikes, the awns were removed from the florets. The spikes were marked with tags mentioning the respective bagging dates indicating different exposure periods of the spikes to airborne fungal inocula.

The florets collected at each sampling time were brought to the laboratory and separated into lemma and grains. The awns were then removed from the individual lemma. The association of fungi with lemma and grains and their percentages of incidence were determined by blotter method according to ISTA rules (Anon., 1996). The lemma and grains were surface sterilized with $1 \%$ chlorox for 2 minutes followed by three times rinsing with sterile distilled water. The lemma and grains were plated separately on three layered moistened blotter papers contained in $9 \mathrm{~cm}$ petridishes. Each plate received 25 lemmas or the same number of grains. The plates with lemma and grains were incubated at $25 \pm 1{ }^{0} \mathrm{C}$ for 7 days under 12/12 hr light and darkness cycle. The incubated lemma and grains were examined under a stereobinocular microscope and the associated fungi were identified following the appropriate keys (Ellis, 1971, 1976; Barnett and Hunter, 1998). When necessary, temporary slides were prepared and identification was done under compound microscope.

The spikes bagged at different days after heading were harvested at the end of dead ripening stage. The harvested spikes were sun-dried and threshed by hands separately for different exposure periods. Black pointed grains were counted by visual observation of grains under a hand lens (10X). Data on number of black pointed grains per spike and percent incidence of black pointed grains were recorded. The grains were indexed for severity of black point infection following 0-5 scale of CIMMYT (Gilchrist, 1985) and the percent black point indices were calculated.

Data on fungal incidence and different parameters of black point infection were analysed statistically following completely randomized design with four replications. The percentage data were transformed following square root transformation method before analysis. The mean values were compared by DMRT.

\section{Results and Discussion}

\section{Association of fungi with floret lemma and grains}

Sixteen different fungi representing 11 genera were detected from the floret lemma and grains of wheat during different stages of grain development. In order of prevalence, the predominant fungi were Alternaria alternata, Bipolaris sorokiniana, Cladosporium cladosporioides, Curvularia lunata, Fusarium spp. and Epicoccum purpurascens. The mean prevalence of the six fungi ranged from 5.57 to $33.68 \%$. Other fungi occurring less commonly were Alternaria triticina, 
Curvularia pallescens, Nigrospora sp., Phoma sp., Chaetomium sp., Bipolaris tetramera, Bipolaris oryzae, Aspergillus flavus, Aspergillus niger and Doratomyces sp. The average incidence of these fungi ranged from 0.04 to $3.22 \%$. The incidence of all the fungi except Fusarium spp. was higher in lemma than in grains. The fungi A. flavus, A. niger and Doratomyces sp. were found associated only with the grains (Table 1 ).

Table 1. Prevalence of fungi associated with lemma and developing grains of wheat.

\begin{tabular}{|c|c|c|c|}
\hline \multirow{2}{*}{ Fungi } & \multicolumn{2}{|c|}{ Prevalence (\%) } & \multirow{2}{*}{ Average (\%) } \\
\hline & Lemma $^{\mathrm{a}}$ & Grains $^{\mathrm{b}}$ & \\
\hline Alternaria alternata & 43.19 & 24.17 & 33.68 \\
\hline Alternaria triticina & 7.31 & 3.83 & 5.57 \\
\hline Aspergillu sfiavus & 0.00 & 0.21 & 0.11 \\
\hline Aspergillus niger & 0.00 & 0.07 & 0.04 \\
\hline Bipolaris oryzae & 0.20 & 0.04 & 0.12 \\
\hline Bipolaris sorokiniana & 32.19 & 27.91 & 30.05 \\
\hline Bipolaris tetramera & 0.60 & 0.38 & 0.49 \\
\hline Chaetomium sp. & 1.09 & 0.52 & 0.81 \\
\hline Cladosporium cladosporioides & 36.29 & 16.67 & 26.48 \\
\hline Curvularia lunata & 19.39 & 10.50 & 14.95 \\
\hline Curvularia pallescens & 3.83 & 2.61 & 3.22 \\
\hline Doratomyces sp. & 0.00 & 0.07 & 0.04 \\
\hline Epicoccum purpurascens & 8.26 & 4.62 & 6.44 \\
\hline Fusarium spp. & 4.94 & 8.33 & 6.64 \\
\hline Nigrospora sp. & 2.63 & 2.29 & 2.46 \\
\hline Phoma sp. & 1.72 & 0.28 & 1.00 \\
\hline
\end{tabular}

${ }^{\mathrm{a}}$ Mean of 32 samplings from heading to dead ripening stage.

${ }^{\mathrm{b}}$ Mean of 28 samplings from seven days after heading to dead ripening stage.

\section{Weekly incidence of fungi on floret lemma}

Weekly determination of fungal population associated with lemma collected from heading to dead ripening stage revealed that the occurrence of different fungi varied with the age of lemma. Infestation of lemma with $A$. alternata, $B$. sorokiniana, C. lunata, E. purpurascens and Fusarium spp. was found to increase with the increasing stages of crop development after heading. In case of $A$. triticina, the incidence was first observed at 7 days after heading, which increased significantly at 14 days but remained statistically similar upto dead ripening stage. The population of C. cladosporioides, on the other hand, increased significantly in the early stages after heading but declined gradually thereafter. However, no specific trend in prevalence was observed in other fungi (Table 2). 
Table 2. Variation in prevalence of fungi associated with lemma of wheat as recorded at weekly intervals beginning from heading to dead ripening stage.

\begin{tabular}{|c|c|c|c|c|c|c|c|c|c|c|c|c|c|}
\hline \multirow{2}{*}{$\begin{array}{c}\text { Days } \\
\text { after } \\
\text { heading }\end{array}$} & \multicolumn{13}{|c|}{ Prevalence of fungi (\%) } \\
\hline & A. a. & A. t. & B. o. & B. s. & B. t. & C. sp. & C. c. & C. 1. & C. p. & E. p. & F. spp. & N. sp. & P. sp. \\
\hline 0 & $\begin{array}{l}5.06 \mathrm{e} \\
(2.35)\end{array}$ & $\begin{array}{l}0.00 c \\
(0.71)\end{array}$ & $\begin{array}{l}0.00 \mathrm{~b} \\
(0.71)\end{array}$ & $\begin{array}{l}0.00 \mathrm{e} \\
(0.71)\end{array}$ & $\begin{array}{l}0.00 \mathrm{~d} \\
(0.71)\end{array}$ & $\begin{array}{l}0.00 \mathrm{c} \\
(0.71)\end{array}$ & $\begin{array}{l}5.31 \mathrm{e} \\
(2.40)\end{array}$ & $\begin{array}{l}4.06 \mathrm{~d} \\
(2.12)\end{array}$ & $\begin{array}{l}0.00 \mathrm{e} \\
(0.71)\end{array}$ & $\begin{array}{l}1.00 d \\
(1.18)\end{array}$ & $\begin{array}{l}1.06 \mathrm{e} \\
(1.20)\end{array}$ & $\begin{array}{l}0.00 \mathrm{c} \\
(0.71)\end{array}$ & $\begin{array}{l}0.0 \mathrm{~d} \\
(0.71)\end{array}$ \\
\hline 7 & $\begin{array}{l}15.25 \mathrm{~d} \\
(3.96)\end{array}$ & $\begin{array}{l}2.31 \mathrm{~b} \\
(1.67)\end{array}$ & $\begin{array}{l}0.00 \mathrm{~b} \\
(0.71)\end{array}$ & $\begin{array}{l}3.56 \mathrm{~d} \\
(2.01)\end{array}$ & $\begin{array}{l}0.00 \mathrm{~d} \\
(0.71)\end{array}$ & $\begin{array}{l}0.50 \mathrm{bc} \\
(0.97)\end{array}$ & $\begin{array}{l}10.00 \mathrm{e} \\
(3.23)\end{array}$ & $\begin{array}{l}7.50 \mathrm{~d} \\
(2.80)\end{array}$ & $\begin{array}{l}1.13 d \\
(1.24)\end{array}$ & $\begin{array}{l}1.00 \mathrm{~cd} \\
(1.23)\end{array}$ & $\begin{array}{l}1.06 \mathrm{e} \\
(1.22)\end{array}$ & $\begin{array}{l}0.00 \mathrm{c} \\
(0.71)\end{array}$ & $\begin{array}{l}0.00 \mathrm{~d} \\
(0.71)\end{array}$ \\
\hline 14 & $\begin{array}{l}35.69 \mathrm{c} \\
(6.00)\end{array}$ & $\begin{array}{l}8.44 a \\
(2.98)\end{array}$ & $\begin{array}{l}0.00 \mathrm{~b} \\
(0.71)\end{array}$ & $\begin{array}{l}14.69 \mathrm{c} \\
(3.89)\end{array}$ & $\begin{array}{l}0.00 \mathrm{~d} \\
(0.71)\end{array}$ & $\begin{array}{l}0.50 \mathrm{bc} \\
(0.93)\end{array}$ & $\begin{array}{l}26.81 d \\
(5.14)\end{array}$ & $\begin{array}{l}15.13 c \\
(3.92)\end{array}$ & $\begin{array}{l}4.00 \mathrm{bc} \\
(2.08)\end{array}$ & & $\begin{array}{l}3.56 \mathrm{~d} \\
(2.01)\end{array}$ & $\begin{array}{l}2.13 b \\
(1.62)\end{array}$ & $\begin{array}{l}2.06 \mathrm{~b} \\
(1.59)\end{array}$ \\
\hline 21 & $\begin{array}{l}43.19 \mathrm{c} \\
(6.59)\end{array}$ & $\begin{array}{l}9.06 \mathrm{a} \\
(3.08)\end{array}$ & $\begin{array}{l}0.63 a \\
(1.05)\end{array}$ & $\begin{array}{l}30.13 \mathrm{~b} \\
(5.53)\end{array}$ & $\begin{array}{l}0.00 \mathrm{~d} \\
(0.71)\end{array}$ & $\begin{array}{l}0.94 a b c \\
(1.19)\end{array}$ & $\begin{array}{l}50.06 \mathrm{bc} \\
(7.09)\end{array}$ & $\begin{array}{l}19.94 b c \\
(4.50)\end{array}$ & $\begin{array}{l}8.13 a \\
(2.90)\end{array}$ & $\begin{array}{l}8.00 \mathrm{~b} \\
(2.90)\end{array}$ & $\begin{array}{l}4.50 \mathrm{~cd} \\
(2.21)\end{array}$ & $\begin{array}{l}2.13 b \\
(1.60)\end{array}$ & $\begin{array}{l}3.00 \mathrm{~b} \\
(1.86)\end{array}$ \\
\hline 28 & $\begin{array}{l}54.94 \mathrm{~b} \\
(7.44)\end{array}$ & $\begin{array}{l}10.19 a \\
(3.26)\end{array}$ & $\begin{array}{l}1.00 \mathrm{a} \\
(1.18)\end{array}$ & $\begin{array}{l}50.13 a \\
(7.09)\end{array}$ & $\begin{array}{l}2.19 a \\
(1.63)\end{array}$ & $\begin{array}{l}0.56 \mathrm{bc} \\
(0.99)\end{array}$ & $\begin{array}{l}69.75 a \\
(8.37)\end{array}$ & $\begin{array}{l}29.94 a \\
(5.49)\end{array}$ & $\begin{array}{l}4.63 b c \\
(2.25)\end{array}$ & $\begin{array}{l}10.00 \mathrm{~b} \\
(3.22)\end{array}$ & $\begin{array}{l}6.25 b c \\
(2.59)\end{array}$ & $\begin{array}{l}4.00 \mathrm{a} \\
(2.11)\end{array}$ & $\begin{array}{l}3.00 \mathrm{~b} \\
(1.86)\end{array}$ \\
\hline 35 & $\begin{array}{l}\text { 62.00ab } \\
(7.90)\end{array}$ & $\begin{array}{l}8.06 \mathrm{a} \\
(2.91)\end{array}$ & $\begin{array}{l}0.00 \mathrm{~b} \\
(0.71)\end{array}$ & $\begin{array}{l}50.94 a \\
(7.16)\end{array}$ & $\begin{array}{l}0.69 \mathrm{c} \\
(1.07)\end{array}$ & $\begin{array}{l}2.19 a b \\
(1.55)\end{array}$ & $\begin{array}{l}\text { 61.19ab } \\
\text { (7.84) }\end{array}$ & $\begin{array}{l}31.00 \mathrm{a} \\
(5.57)\end{array}$ & $\begin{array}{l}2.75 c \\
(1.79)\end{array}$ & $\begin{array}{l}8.19 b \\
(2.94)\end{array}$ & $\begin{array}{l}7.50 \mathrm{ab} \\
(2.82)\end{array}$ & $\begin{array}{l}5.56 a \\
(2.44)\end{array}$ & $\begin{array}{l}4.56 a \\
(2.23)\end{array}$ \\
\hline 42 & $\begin{array}{l}70.00 \mathrm{a} \\
(8.38)\end{array}$ & $\begin{array}{l}10.00 \mathrm{a} \\
(3.23)\end{array}$ & $\begin{array}{l}0.00 \mathrm{~b} \\
(0.71)\end{array}$ & $\begin{array}{l}56.06 \mathrm{a} \\
(7.51)\end{array}$ & $\begin{array}{l}0.44 c d \\
(0.95)\end{array}$ & $\begin{array}{l}1.50 \mathrm{ab} \\
(1.35)\end{array}$ & $\begin{array}{l}41.88 \mathrm{c} \\
(6.49)\end{array}$ & $\begin{array}{l}\text { 22.38abc } \\
(4.76)\end{array}$ & $\begin{array}{l}4.63 \mathrm{bc} \\
(2.25)\end{array}$ & $\begin{array}{l}11.44 \mathrm{~b} \\
(3.43)\end{array}$ & $\begin{array}{l}\text { 5.56bcd } \\
(2.45)\end{array}$ & $\begin{array}{l}5.00 \mathrm{a} \\
(2.34)\end{array}$ & $\begin{array}{l}1.13 c \\
(1.27)\end{array}$ \\
\hline 49 & $\begin{array}{l}\text { 59.38ab } \\
(7.72)\end{array}$ & $\begin{array}{l}10.38 a \\
(3.28)\end{array}$ & $\begin{array}{l}0.00 \mathrm{~b} \\
(0.71)\end{array}$ & $\begin{array}{l}52.00 \mathrm{a} \\
(7.23)\end{array}$ & $\begin{array}{l}1.50 \mathrm{~b} \\
(1.40)\end{array}$ & $\begin{array}{l}2.50 \mathrm{a} \\
(1.73)\end{array}$ & $\begin{array}{l}25.31 d \\
(5.06)\end{array}$ & $\begin{array}{l}25.19 \mathrm{ab} \\
(5.04)\end{array}$ & $\begin{array}{l}5.38 \mathrm{ab} \\
(2.40)\end{array}$ & $\begin{array}{l}23.94 a \\
(4.92)\end{array}$ & $\begin{array}{l}10.00 \mathrm{a} \\
(3.22)\end{array}$ & $\begin{array}{l}2.25 b \\
(1.64)\end{array}$ & $\begin{array}{l}0.00 \mathrm{~d} \\
(0.71)\end{array}$ \\
\hline $\mathrm{CV}(\%)$ & 7.20 & 9.66 & 17.34 & 8.82 & 15.57 & 32.32 & 11.16 & 13.28 & 17.83 & 12.88 & 13.37 & 14.00 & 14.03 \\
\hline
\end{tabular}

Means within a column followed by a common letter do not differ significantly $(\mathrm{p}=0.05)$.

Data within parentheses are square root $(\mathrm{x}+0.5)$ transformed values.

A. а. = A. alternata, A. t. = A. triticina, B. o. = B. oiyzae, B. s. = B. sorokiniana, B. t. = B. tetramera, C. sp. $=$ Chaetomium sp., C. c. $=$ C. cladosporioides, C. 1. = C. lunata, C. p. = C. pallescens, D. sp. = Doratomyces sp., E. p. = E. purpurascens, F. spp. = Fusarium spp., N. sp. = Nigrospora sp., P. sp. = Phoma sp. 
Table 3. Variation in prevalence of fungi associated with developing grains of wheat as recorded at weekly intervals beginning from seven days after heading to dead ripening stage.

\begin{tabular}{|c|c|c|c|c|c|c|c|c|c|c|c|c|c|c|c|c|}
\hline \multirow{2}{*}{$\begin{array}{c}\text { Days } \\
\text { after } \\
\text { heading }\end{array}$} & \multicolumn{16}{|c|}{ Prevalence of fungi (\%) } \\
\hline & A. a. & A. t. & A. $f$. & A. $n$. & B. o. & B. s. & B. t. & C. sp. & C. c. & C. 1. & C. p. & D. sp. & E. p. & F. spp. & N. sp. & P. sp. \\
\hline \multirow[t]{2}{*}{7} & 5.31c & $1.31 \mathrm{e}$ & $0.00 \mathrm{~b}$ & $0.00 \mathrm{~b}$ & $0.00 \mathrm{~b}$ & $1.00 \mathrm{~d}$ & $0.00 \mathrm{~b}$ & $0.44 \mathrm{ab}$ & $3.56 \mathrm{~d}$ & $4.00 \mathrm{e}$ & $0.56 \mathrm{e}$ & $0.00 \mathrm{~b}$ & $0.00 \mathrm{~d}$ & $1.19 \mathrm{~d}$ & $0.00 c$ & $0.00 \mathrm{c}$ \\
\hline & (2.39) & $(1.34)$ & $(0.71)$ & $(0.71)$ & $(0.71)$ & $(1.23)$ & $(0.71)$ & $(0.92)$ & $(2.00)$ & $(2.11)$ & $(1.01)$ & $(0.71)$ & $(0.71)$ & $(1.28)$ & $(0.71)$ & $(0.71)$ \\
\hline \multirow[t]{2}{*}{14} & $14.13 \mathrm{~b}$ & 4.00bcd & $0.00 \mathrm{~b}$ & $0.00 \mathrm{~b}$ & $0.00 \mathrm{~b}$ & $7.25 \mathrm{c}$ & $0.00 \mathrm{~b}$ & $0.00 \mathrm{~b}$ & $6.38 \mathrm{~cd}$ & 6.06de & $3.50 \mathrm{ab}$ & $0.00 \mathrm{~b}$ & $0.00 \mathrm{~d}$ & $6.56 c$ & $1.94 \mathrm{~b}$ & $0.00 \mathrm{c}$ \\
\hline & $(3.76)$ & $(2.12)$ & $(0.71)$ & $(0.71)$ & $(0.71)$ & $(2.76)$ & $(0.71)$ & $(0.71)$ & $(2.61)$ & $(2.55)$ & (1.99) & $0.71)$ & $(0.71)$ & $(2.65)$ & (1.54) & $(0.71)$ \\
\hline \multirow[t]{2}{*}{21} & $19.63 \mathrm{~b}$ & $5.06 \mathrm{ab}$ & $0.00 \mathrm{~b}$ & $0.00 \mathrm{~b}$ & $0.00 \mathrm{~b}$ & $22.88 b$ & $0.00 \mathrm{~b}$ & $0.56 a b$ & $25.00 \mathrm{a}$ & $8.19 \mathrm{~cd}$ & $4.94 a$ & $0.00 \mathrm{~b}$ & $3.88 c$ & $7.56 \mathrm{bc}$ & 3.06ab & $0.00 \mathrm{c}$ \\
\hline & $(4.46)$ & (2.35) & $(0.71)$ & $(0.71)$ & $(0.71)$ & $(4.82)$ & $(0.71)$ & $(0.99)$ & $(5.03)$ & (2.93) & (2.32) & $(0.71)$ & (2.09) & $(2.82)$ & (1.88) & $(0.71)$ \\
\hline \multirow[t]{2}{*}{28} & $29.56 a$ & 4.50abc & $0.00 \mathrm{~b}$ & $0.00 \mathrm{~b}$ & $0.31 \mathrm{a}$ & $38.31 \mathrm{a}$ & $1.00 \mathrm{a}$ & $0.00 \mathrm{~b}$ & $31.06 \mathrm{a}$ & $10.88 b c$ & $2.56 \mathrm{bc}$ & $0.00 \mathrm{~b}$ & 7.44ab & $9.8 \mathrm{lbc}$ & $2.44 \mathrm{ab}$ & $0.56 \mathrm{ab}$ \\
\hline & $(5.45)$ & $(2.22)$ & $(0.71)$ & $(0.71)$ & $(0.88)$ & $(6.18)$ & (1.23) & $(0.71)$ & $(5.60)$ & (3.37) & $(1.72)$ & $(0.71)$ & $(2.80)$ & (3.19) & (1.71) & (1.02) \\
\hline \multirow[t]{2}{*}{35} & $33.44 a$ & $5.50 \mathrm{a}$ & $0.00 \mathrm{~b}$ & $0.00 \mathrm{~b}$ & $0.00 \mathrm{~b}$ & $40.00 \mathrm{a}$ & $0.25 b$ & $1.25 a$ & $26.06 a$ & $12.50 \mathrm{ab}$ & $1.25 \mathrm{de}$ & $0 \mathrm{~b}$ & $5.56 \mathrm{~b}$ & 10.69ab & 2.94ab & $1.00 \mathrm{a}$ \\
\hline & $(5.80)$ & $(2.44)$ & $(0.71)$ & $(0.71)$ & $(0.71)$ & 3) & $(0.84)$ & 26) & $(5.14)$ & 9) & (1 & $(C$ & 5) & 3) & 34) & $(1.20)$ \\
\hline \multirow[t]{2}{*}{42} & $35.13 a$ & $3.00 \mathrm{~d}$ & $0.00 \mathrm{~b}$ & $0.00 \mathrm{~b}$ & $0.00 \mathrm{~b}$ & 40.94a & $0.25 b$ & $0.25 a b$ & $15.50 \mathrm{~b}$ & $15.81 \mathrm{a}$ & 2.06cd & $0.00 \mathrm{~b}$ & 7.44ab & $8.56 \mathrm{bc}$ & 3.56a & $0.38 \mathrm{bc}$ \\
\hline & (5.93) & $(2.86)$ & $(0.71)$ & $(0.71)$ & $(0.71)$ & $(6.42)$ & $(0.84)$ & $(0.84)$ & (3.98) & (4. & (1 & 71) & 31) & (3 & $(2.00)$ & $(0.92)$ \\
\hline \multirow[t]{2}{*}{49} & $32.00 \mathrm{a}$ & $3.44 \mathrm{~cd}$ & $1.50 \mathrm{a}$ & $0.50 a$ & $0.00 \mathrm{~b}$ & $45.00 \mathrm{a}$ & $1.13 a$ & 1.l3ab & $9.13 c$ & $16.06 a$ & 3.38ab & $0.50 \mathrm{a}$ & $8.00 \mathrm{a}$ & $13.94 a$ & $2.06 \mathrm{~b}$ & $0.00 \mathrm{c}$ \\
\hline & (5.69) & (1.98) & (1.40) & $(1.00)$ & $(0.71)$ & 2) & (1.26) & (1.23) & (3.09) & 3) & (1.97) & $(1.00)$ & $(2.90)$ & (3.78) & (1.59) & $(0.71)$ \\
\hline CV(\%) & 11.65 & 9.65 & 9.64 & 5.20 & 12.62 & 12.90 & 17.54 & 33.45 & 10.63 & 12.25 & 13.24 & 5.20 & 11.88 & 11.96 & 13.99 & 17.92 \\
\hline
\end{tabular}

Means within a column followed by a common letter do not differ significantly ( $\mathrm{p}=0.05)$.

Data within parentheses are square root $(x+0.5)$ transformed values.

A. а. $=$ A. alternata, A. t. $=$ A. triticina, A. f. $=$ A. flavus, A. n. $=$ A. niger, B. o. $=$ B. oryzae, B. s. $=$ B. sorokiniana, B. t. $=$ B. tetramera, C. sp. = Chaetomium sp., C. c. = C. cladosporioides, C. $1 .=$ C. lunata, C. p. = C. pallescens, D. sp. = Doratomyces sp., E. p. = E. purpurascens, F. spp. = Fusarium spp., N. sp. = Nigrospora sp., P. sp. $=$ Phoma sp. 


\section{Weekly incidence of fungi on developing grains}

The incidence patterns of A. alternata, B. sorokiniana, C. lunata, E. purpurascens and Fusarium spp. on developing grains were almost similar to those as observed on the floret lemma. A gradual increasing trend in population of these fungi was observed as the grain approached towards dead ripening stage. Seedborne incidence of $A$. triticina and $C$. cladosporioides increased in the early stages but declined significantly in the later stages of grain development. The other fungi did not show any definite pattern in their weekly prevalence on the developing grains (Table 3 ).

\section{Incidence of black point on wheat grains}

Occurrence of black point infection in wheat grains varied with the variation in exposure periods of the spikes to airborne inocula of the fungi after heading to dead ripening stage. A gradual increasing trend in number of black pointed grains per spike, percent black pointed grains and percent black point index was observed with the increase in exposure period of the spikes in days after heading (DAH). Number of black pointed grains per spike ranged from 0.56 to 8.74 depending on the length of exposure period. The lowest number of black pointed grains per spike was recorded under 0 day exposure after heading, which was statistically similar to the exposure period of 7 DAH. Exposure of spikes for 14 DAH caused significant increase in number of black pointed grains per spike as compared to those recorded under the expoure periods of 0 and $7 \mathrm{DAH}$, and the increasing trend remained statistically similar upto the expoure of $35 \mathrm{DAH}$. The highest number of black pointed grains per spike was recorded when the spikes were exposed for $49 \mathrm{DAH}$, which was statistically similar to exposure of 42 DAH but significantly higher as compared to other exposure periods. Almost similar increasing trend was observed in case of percent black pointed grains. The lowest incidence of $1.31 \%$ black pointed grains was observed under the exposure of $0 \mathrm{DAH}$, while the highest of $21.50 \%$ was recorded at the exposure of $49 \mathrm{DAH}$. The percentages of black pointed grains recorded at the exposures of 49 and $42 \mathrm{DAB}$ were statistically similar to one another but significantly higher than those recorded under the other exposure periods. Percent black point index was found maximum when the spikes were exposed for $49 \mathrm{DAH}$, and it was statistically similar to the exposure of $42 \mathrm{DAH}$. The lowest black point index was recorded at the exposure of $0 \mathrm{DAH}$, which was significantly lower compared to other periods of exposure (Table 4).

In the present investigation, altogether 16 fungi belonging to 11 genera were found to be associated with lemma and developing grains of wheat during the period from heading to dead ripening stage. The most frequently occurring fungi were $A$. alternata and $B$. sorokiniana. The incidence of total fungi increased with the increase in days from heading to dead ripening stage. Association of these 
fungi with wheat grains were also reported by other researchers (Ali and Fakir, 1992; Bazlur Rashid et al., 1995; Mahmud, 2005). Magan and Lacey (1986) reported that the population of A. alternata, Cladosporium spp., Fusarium spp. and E. nigrum increased rapidly between anthesis and harvest. Talukder and Fakir (1991) found that A. tenuis occurred most frequently at full ripe stage and $E$. purpurascens at dead ripe stage, whereas $C$. cladosporioides was the most prevalent fungus between flowering and anthesis, and between soft and hard dough stages.

Table 4. Incidence of black point disease of wheat grains under different exposure periods of the spikes to airborne inocula of the causal fungi beginning from heading to dead ripening stage.

\begin{tabular}{l|l|l|l}
\hline $\begin{array}{c}\text { Exposure periods of spikes } \\
\text { (days after heading) }\end{array}$ & $\begin{array}{c}\text { Black pointed } \\
\text { grains/spike }\end{array}$ & $\begin{array}{c}\text { Percent black } \\
\text { pointed grains }\end{array}$ & $\begin{array}{c}\text { Percent black } \\
\text { point index }\end{array}$ \\
\hline 0 & $0.56 \mathrm{~d}$ & $1.31 \mathrm{c}(1.32)$ & $0.63 \mathrm{e}(1.05)$ \\
7 & $1.34 \mathrm{~d}$ & $3.19 \mathrm{c}(1.89)$ & $1.65 \mathrm{~d}(1.45)$ \\
14 & $4.69 \mathrm{c}$ & $10.88 \mathrm{~b}(3.36)$ & $6.18 \mathrm{c}(2.56)$ \\
21 & $5.75 \mathrm{c}$ & $12.31 \mathrm{~b}(3.57)$ & $6.89 \mathrm{c}(2.72)$ \\
28 & $6.16 \mathrm{c}$ & $14.75 \mathrm{~b}(3.88)$ & $7.51 \mathrm{c}(2.83)$ \\
35 & $6.69 \mathrm{bc}$ & $15.25 \mathrm{~b}(3.94)$ & $7.84 \mathrm{bc}(2.88)$ \\
42 & $8.26 \mathrm{ab}$ & $21.06 \mathrm{a}(4.62)$ & $9.79 \mathrm{ab}(3.20)$ \\
49 & $8.74 \mathrm{a}$ & $21.50 \mathrm{a}(4.66)$ & $9.98 \mathrm{a}(3.23)$ \\
\hline $\mathrm{CV}(\%)$ & 23.81 & 13.21 & 8.97 \\
\hline
\end{tabular}

Means within a column followed by a common letter do not differ significantly $(p=0.05)$. Data within parentheses are square root $(x+0.5)$ transformed values.

Visual observation of wheat grains after harvest indicated that black point incidence increased with the increase in exposure period of the spikes to airborne inocula of the causal fungi. The increase in black point incidence might be due to increasing prevalence of black point fungi occurred between heading and dead ripening stage. Ahmed (1989) reported that black point symptom appeared at the late stage of dough development and became conspicuous towards ripening of the grain. In a similar study, Talukder and Fakir (1993) observed that the disease appeared on the floret grains at hard dough stage and it became pronounced at the dead ripening stage. From the results of the present study, it may be concluded that the population of fungi associated with floret lemma and developing grains of wheat increased with their age and the subsequent incidence of black point disease increased with the exposure period of the spikes to airborne inocula of the fungi. 


\section{References}

Adlakha, K.L. and L.M. Joshi. 1974. Black point of wheat. Indian Phytopath. 27: 41-44.

Ahmed, D.N. 1989. Mycoflora associated with developing wheat grains. M.Sc.(Ag.) Thesis. Department of Plant Pathology, Bangladesh Agricultural University, Mymensingh. 50 p.

Ahmed, D.N., A.L. Khan, B. Meah and M.A.T. Mia. 1994. An investigation to mycoflora associated with developing wheat grains. Ann. Bangladesh Agric. 4(2): 95-100.

Ali, M.H. and G.A. Fakir. 1992. Fungi associated with wheat grains in Bangladesh and their pathogenic significance. Bangladesh J. Bot. 21(2): 173-180.

Anonymous. 1996. International Rules for Seed Testing. International Seed Testing Association (ISTA). Seed Sci. \& Tech. 24 (supplement): 29-72.

Barnett, H.L. and B.B. Hunter. 1998. Illustrated genera of Imperfect Fungi. Fourth Edition, APS Press, 3340 Pilot Knob Road, St. Paul, Minnesota, USA. 218 p.

Bazlur Rashid, A.Q.M., G.A. Fakir, M.A.T. Mia and K.S. Uddin. 1995. Seed health studies and crop production in Bangladesh. J. Sustainable Agric. 6(2/3): 93-122.

Dey, T.K., N. Chowdhury, A. Ayub and B.K. Goswami. 1992. Black point of wheat: Occurrence, effect of fungicidal seed treatment on germination and quality characters. Bangladesh J. Bot. 22(1): 27-32.

Dhruj, I.U. 1991. Studies on black point of wheat. Ph.D. Thesis. Division of Mycology and Plant Pathology, Indian Agricultural Research Institute, New Delhi. 77 p.

Ellis, M.B. 1971. Dematiaceous Hyphomycetes. CMI, Kew, Surrey, England. 608 p.

Ellis, M.B. 1976. More Dematiaceous Hyphomycetes. CMI, Kew, Surrey, England. 507 p.

Fakir, G.A. 1998. Black point disease of wheat in Bangladesh. Second Edition. Seed Pathology Laboratory, Bangladesh Agricultural University, Mymensingh. 81 p.

Fakir, G.A., M.H. Rahman and G.M.M. Rahman. 1989. Survey on the prevalence of black point fungi of wheat in Bangladesh. Bangladesh J. Plant Pathol. 5(1\&2): 19-29.

Gilchrist, L.I. 1985. CIMMYT methods for screening wheat for Helminthosporium sativum resistance. In: Wheat for More Tropical environments: A Proceeding of the International Symposium. Mexico, D.F.: CIMMYT. pp. 149-15 1.

Khanum, M., Y. Nigar and A.K. Khanzada. 1987. Effect of black point disease on the germination of wheat varieties. Pakistan J. Agril. Res. 8(4): 467-473.

Mahmud, J.A. 2005. Health status of wheat seeds collected from the farmers of Rangpur district. M.S. Thesis. Department of Plant Pathology, Bangabandhu Sheikh Mujibur Rahman Agricultural University, Gazipur. 52 p.

Magan, N. and J. Lacey. 1986. The phylloplane mycoflora of ripening wheat and effect of late fungicide applications. Ann. Appl. Biol. 109(1): 117-128.

Mathur, S.B. and Barry M. Cunfer. 1993. Seedborne diseases and seed health testing of wheat. Danish Government Institute of Seed Pathology for Developing Countries, Denmark. 168 p.

Neergaard, P. 1979. Seed Pathology. Vol. I. The Macmillan Press Ltd., London and Basingstoke. 839 p. 
Rahman, G.M.M. and M.R. Islam. 1998. Effect of black point of wheat on some qualitative characters of its grain and seed vigour. Bangladesh J. Agril. Res. 23(2): 283-287.

Talukder, K.A. and G.A. Fakir. 1991. Association of fungi with spikelet parts during development of wheat. Fourth Biennial Conf., Bangladesh Phytopathol. Soc. Abstr. No. 40.

Talukder, K.A. and G.A. Fakir. 1993. Occurrence of black point and black point fungi in developing grain of wheat. Fifth Biennial Conf., Bangladesh Phytopathol. Soc., Bangladesh Agricultural University, Mymensingh. pp. 91-92. 